\title{
Dynamical evolution of C/2017 K2 PANSTARRS
}

\author{
Małgorzata Królikowska ${ }^{1}$ and Piotr A. Dybczyński ${ }^{2}$ \\ 1 Space Research Centre of Polish Academy of Sciences, Bartycka 18A, Warszawa, Poland \\ e-mail: mkr@cbk. waw.pl \\ 2 Astronomical Observatory Institute, Faculty of Physics, Adam Mickiewicz University, Słoneczna 36, Poznań, Poland \\ e-mail: dybol@amu.edu.pl
}

Received 28 February 2018 / Accepted 9 April 2018

\begin{abstract}
Context. The comet C/2017 K2 PANSTARRS drew attention to its activity at the time of its discovery in May 2017 when it was about 16 au from the Sun. This Oort spike comet will approach its perihelion in December 2022, and the question about its dynamical past is an important issue to explore.

Aims. In order to answer the question of whether C/2017 K2 is a dynamically old or new comet it is necessary to obtain its precise osculating orbit, its original orbit, and propagate its motion backwards in time to the previous perihelion. Knowledge of the previous perihelion distance is necessary to distinguish between these two groups of the Oort spike comets. We have studied the dynamical evolution of $\mathrm{C} / 2017 \mathrm{~K} 2$ to the previous perihelion (backward calculations for about 3-4 Myr) as well as to the future (forward calculations for about $0.033 \mathrm{Myr}$ ) using the swarm of virtual comets (VCs) constructed from a nominal osculating orbit of this comet which we determined here using all positional measurements available at the moment. Outside the planetary system both Galactic and stellar perturbations were taken into account.

Results. We derive that $\mathrm{C} / 2017 \mathrm{~K} 2$ is a dynamically old Oort spike comet $\left(1 / a_{\text {prev }}=(48.7 \pm 7.9) \times 10^{-6} \mathrm{au}^{-1}\right)$ with the previous perihelion distance below 10 au for $97 \%$ of VCs (nominal $q_{\text {prev }}=3.77 \mathrm{au}$ ). According to the present data this comet will be perturbed into a more tightly bound orbit after passing the planetary zone $\left(1 / a_{\text {fut }}=(1140.4 \pm 8.0) \times 10^{-6} \mathrm{au}^{-1}, q_{\mathrm{fut}}=1.79336 \pm 0.00006 \mathrm{au}\right)$ provided that non-gravitational effects will not change the orbit significantly.

Conclusions. C/2017 K2 has already visited our planetary zone during its previous perihelion passage. Thus, it is almost certainly a dynamically old Oort spike comet. The future orbital solution of this comet is formally very precise, however, it is much less definitive since the presented analysis is based on pre-perihelion data taken at very large heliocentric distances (23.7-14.6 au from the Sun), and this comet can experience a significant non-gravitational perturbation during the upcoming perihelion passage in 2022.
\end{abstract}

Key words. comets: individual: C/2017 K2 - Oort Cloud - celestial mechanics

\section{Introduction}

This short analysis was inspired by a very interesting study of the activity and past dynamical motion of comet C/2017 K2 PANSTARRS recently published by Hui et al. (2018). Here, we have focussed on dynamical aspects of their paper. The authors examine a history of $\mathrm{K} 2{ }^{1}$ for $1 \mathrm{Myr}$ taking into account planetary perturbations, relativistic corrections and perturbations from a Galactic tide. At the epoch of $1 \mathrm{Myr}$ in the past they obtained $1 / a \simeq(36.1 \pm 17.1) \times 10^{-6} \mathrm{au}^{-1}$ and using this value alone they try to deduce whether a comet is dynamically new or old. This value of $1 / a$ places $\mathrm{K} 2$ in an area of the Oort spike region where dynamically new and dynamically old comets coexist (Królikowska \& Dybczyński 2017). Therefore, Hui et al. (2018) concluded that the dynamical status of $\mathrm{K} 2$ "must be regarded as unsettled" since they stopped the integration more or less in the middle of the time interval to the previous perihelion. This conclusion drew our attention to K2. In the following, we discuss the dynamical evolution of $\mathrm{K} 2$ backwards to the previous perihelion and forwards to the subsequent one.

\section{Observational material}

The observational material was retrieved on 2018 February 21 from the Minor Planet Center. It consists of 450 positional

1 We use "K2" as an abbreviation for $\mathrm{C} / 2017 \mathrm{~K} 2$, as in Hui et al. (2018). measurements covering a period from 2013 May 12 to 2018 January 23. This time interval of data is four months longer than in Hui et al. (2018), which means, however, that it is longer only by seven per cent.

The positional observations are distributed heterogeneously over the studied period. K2 was discovered on 21 May 2017 by the Panoramic Survey Telescope and Rapid Response System (Pan-STARRS) in Hawaii when it was 16.1 au from the Sun. Later, almost twenty pre-discovery measurements dating back to 2013 May 12 were found. However, one large gap of about $2.5 \mathrm{yr}$ and two shorter gaps of about six months and eight months still exist inside this pre-discovery interval of measurements. Since its discovery the comet has been observed continuously. The distribution of positional observations as well as heliocentric and geocentric distance changes of $\mathrm{K} 2$ are shown in Fig. 1. It is worth mentioning here that such a range of astrometric cometary positions is almost optimal for the past dynamical history investigations due to almost five years of its data interval and the lack of non-gravitational effects at such a large heliocentric distance.

\section{Observed, original, and future orbit of K2}

We determined the osculating orbit (hereafter nominal orbit) from all positional measurements available in the MPC database ${ }^{2}$ on 2018 February 21. Details of selection

\footnotetext{
2 http://wWW.minorplanetcenter.net/db_search
} 


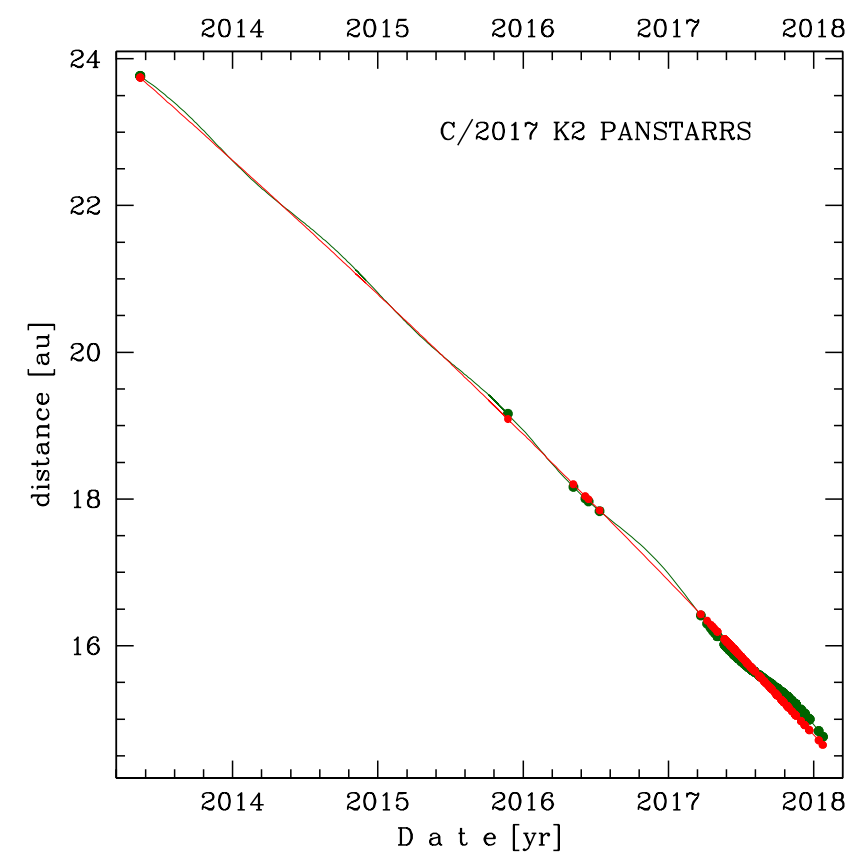

Fig. 1. Heliocentric (thin red curve) and geocentric (green curve) distance of K2 during the time interval given in Table 1 . The time distribution of positional observations of $\mathrm{K} 2$ with corresponding heliocentric distance at which they were taken is shown with red dots. The geocentric distances of measurements are shown with green dots but they are almost covered by red dots because of similarly large distances from the Sun and the Earth.

and weighting of positional data are described in Królikowska \& Dybczyński (2010).

We present here two solutions. The first (A1) is based on weighted data (second column of Table 1) and we treated it as the preferred solution. For comparison, we also present the solution based on unweighted data (solution A0, third column of Table 1) to show the magnitude of orbital element dispersions at the important stages of a dynamical evolution of $\mathrm{K} 2$ : at $250 \mathrm{au}$ from the Sun (original and future orbits), and at the moments of a previous and subsequent perihelion (previous and subsequent orbit). Our two solutions are consistent with each other within about $1 \sigma$ combined error and also with the JPL solution based on the same time interval (last column of Table 1; it is important to stress that we used a different selection and weighting scheme for data than that used by JPL).

Hui et al. (2018) notice that K2 activity has been slowly increasing since 2013. However, no one has ever been able to derive a reliable non-gravitational orbit for a comet that is so far away from the Sun, even assuming other forms of a $g(r)$-like function (see Marsden et al. (1973)) compatible with a CO-sublimation. We have checked, and found that it is not possible in this case either (because the non-gravitational effects are too weak).

Next, in order to control the uncertainties in the motion of $\mathrm{K} 2$ at each stage of our dynamical calculations, we cloned its orbit and constructed a swarm of 5000 VCs resembling the observations (using the method described by Sitarski 1998). This allowed us to present the uncertainties of all orbital elements for dynamically evolved orbits, in other words, original and future orbits at 250 au from the Sun when planetary perturbations are negligible. These orbits are presented in Table 2. Values of $1 / a_{\text {ori }}$ for both our solutions are greater than that obtained by Hui etal. (2018). At these steps of dynamical evolution of $\mathrm{K} 2$ the equations of motion have been integrated using the recurrent power series method (Sitarski 1989), taking into account perturbations by all planets and including relativistic effects.

\section{4. $\mathrm{K} 2$ in its previous perihelion}

At a distance beyond 250 au from the Sun we can ignore planetary perturbations, and study the earlier dynamical evolution of $\mathrm{K} 2$ until it reaches its previous perihelion taking into account the perturbations from the Galactic disk, Galactic centre and all known potentially perturbing stars. The list of the ten potentially strongest stellar perturbers of K2's past motion is presented in Table 4.

We have used here the same model of Galactic gravitational potential as in Królikowska \& Dybczyński (2017). We updated only the list of known passing stars. This was a consequence of recently published results of Gaia (Gaia Collaboration 2016) and RAVE (Kunder et al. 2017). Ranges of selected orbital elements of $\mathrm{K} 2$ at the previous perihelion are shown in Table 3, and the swarm of VCs projected onto the $1 / a-q$ plane for the basic solution A1 is shown in the left panel of Fig. 2. One can see that a small percentage of VCs formed a dispersed wing stretching towards the upper left corner of this plot (large $q_{\text {prev }}$, small $\left.1 / a_{\text {prev }}\right)$. This wing is so long that to keep a reasonable vertical scale we have omitted two VCs with extremely large values of $q_{\text {prev }}\left(473 \mathrm{au}\right.$ and $3037 \mathrm{au}$, both at $1 / a_{\text {prev }}=22 \times 10^{-6} \mathrm{au}^{-1}$ ). As a result of this dispersion the $q_{\text {prev }}$-distribution is far from Gaussian. This basic solution (A1) gives the nominal previous perihelion of $q_{\text {prev }}=3.815$ au and $97 \%$ of VCs with $q_{\text {prev }}<10$ au which means that we can conclude that $\mathrm{K} 2$ is a dynamically old comet which passed its previous perihelion about $3 \mathrm{Myr}$ ago deep inside the planetary zone.

\section{K2 in the subsequent perihelion}

We study the future motion of $\mathrm{K} 2$ to the subsequent perihelion in a similar way as above. Orbital elements of $\mathrm{K} 2$ at the subsequent perihelion are shown in Table 3, whereas a full swarm of VCs projected onto the $1 / a-q$ plane for preferred solution A1 is shown in the right panel of Fig. 2. It turns out that the swarm of VCs at the subsequent perihelion is very compact and gives precise values of orbital elements. According to our calculations $\mathrm{K} 2$ will pass subsequent perihelion at the distance of about $1.8 \mathrm{au}$, similarly as in the current passage, and its orbital period after leaving the planetary zone will be of only about $32500 \mathrm{yr}$ (Table 3). We would like to stress, however, that the future dynamical evolution of $\mathrm{K} 2$ can be calculated in a certain way only after the comet passing through the perihelion, because non-gravitational effects can significantly change current predictions.

\section{Overall evolution during three perihelion passages}

The continuous dynamical evolution of K2 starting at the previous perihelion, through the observed one, up to the subsequent perihelion passage is depicted in Fig. 3. In both panels the left vertical axis is expressed in au and corresponds to the perihelion distance plot ( $q$, green lines) as well as the heliocentric distance plots ( $r$, thin, vertical blue lines). The right vertical axis is expressed in degrees and describes the evolution of the osculating inclination ( $i$, magenta lines) and the argument of perihelion 
Table 1. Osculating heliocentric orbit of K2 based on 450 positional measurements spanning the time interval from 2013 May 12 to 2018 January 23 available at MPC on 2018 February 21.

\begin{tabular}{lccc}
\hline \hline & Solution A1 & Solution A0 & JPL solution \\
\hline Perihelion distance [au] & $1.81086204 \pm 0.00005500$ & $1.81081861 \pm 0.00006434$ & $1.81072035 \pm 0.00013422$ \\
Eccentricity & $1.00032653 \pm 0.00001443$ & $1.00031764 \pm 0.00001823$ & $1.00029212 \pm 0.00003797$ \\
Time of perihelion passage [TT] & $20221221.412478 \pm 0.030195$ & $20221221.420049 \pm 0.041069$ & $20221221.465966 \pm 0.085538$ \\
Inclination [deg] & $87.553729 \pm 0.000023$ & $87.553662 \pm 0.000045$ & $87.553645 \pm 0.000095$ \\
Argument of perihelion [deg] & $236.021084 \pm 0.001710$ & $236.022156 \pm 0.002056$ & $236.025192 \pm 0.004281$ \\
Longitude of the ascending node [deg] & $88.181668 \pm 0.000236$ & $88.182090 \pm 0.000461$ & $88.182237 \pm 0.000973$ \\
Epoch of osculation [TT] & 20170705 & 20170705 & 20170705 \\
RMS & 0.38 arcsec & 0.47 arcsec & 0.52 (normalized) \\
\hline
\end{tabular}

Notes. Ecliptic of J2000 is used. Solution A1 is based on weighted measurements whereas A0 is based on unweighted data. For comparison, Jet Propulsion Laboratory (JPL) solution is presented in the last column; it is based on 446 observations and the same interval of data (retrieved from the JPL Small-Body Database Browser on 21 February 2018).

Table 2. Original and future barycentric orbits of $\mathrm{K} 2$ at $250 \mathrm{au}$ from the Sun, that is, before entering and after leaving the planetary zone, respectively.

\begin{tabular}{|c|c|c|c|c|}
\hline & \multicolumn{2}{|c|}{ Solution A1 } & \multicolumn{2}{|c|}{ Solution A0 } \\
\hline & Original orbit & Future orbit & Original orbit & Future orbit \\
\hline Perihelion distance $[\mathrm{au}]$ & $1.79551272 \pm 0.00005475$ & $1.79335881 \pm 0.00005584$ & $1.79546949 \pm 0.00006519$ & $1.79331479 \pm 0.00006646$ \\
\hline Eccentricity & $0.99991296 \pm 0.00001423$ & $0.99795491 \pm 0.00001431$ & $0.99990416 \pm 0.00001821$ & $0.99794611 \pm 0.00001833$ \\
\hline Inverse of the semimajor axis & $48.48 \pm 7.93$ & $1140.4 \pm 8.0$ & $53.38 \pm 10.15$ & $1145.3 \pm 10.3$ \\
\hline Time of perihelion passage $[\mathrm{TT}]$ & $20221218.855081 \pm 0.029861$ & $20221219.872785 \pm 0.030085$ & $20221218.862555 \pm 0.041019$ & $20221219.880427 \pm 0.041289$ \\
\hline Inclination & $87.573197 \pm 0.000023$ & $87.789103 \pm 0.000017$ & $87.573129 \pm 0.000045$ & $87.789042 \pm 0.000042$ \\
\hline Argument of perihelion & $236.214199 \pm 0.001702$ & $236.051977 \pm 0.001691$ & $236.215269 \pm 0.002075$ & $236.053032 \pm 0.002063$ \\
\hline Longitude of the ascending node & $88.086028 \pm 0.000236$ & $88.254521 \pm 0.000240$ & $88.086450 \pm 0.000460$ & $88.254950 \pm 0.000464$ \\
\hline Epoch of osculation $[\mathrm{TT}]$ & 17221020 & 23360713 & 17221020 & 23360822 \\
\hline
\end{tabular}

Notes. Inverse semimajor axis values are in units of $10^{-6} \mathrm{au}^{-1}$, and angular orbital elements are given in degrees.

Table 3. Barycentric orbit elements of $\mathrm{K} 2$ at the previous and subsequent perihelion for two considered solutions A1 and A0.

\begin{tabular}{lcccc}
\hline \hline & \multicolumn{2}{c}{ Solution A1 } & \multicolumn{2}{c}{ Solution A0 } \\
\cline { 2 - 5 } & Previous orbit & Subsequent orbit & Previous orbit & Subsequent orbit \\
\hline Perihelion distance $[\mathrm{au}]$ & $2.625: 3.815: 8.335$ & $1.793346 \pm 0.000056$ & $2.264: 3.041: 6.391$ & $1.793300 \pm 0.000067$ \\
Aphelion distance $\left[10^{3} \mathrm{au}\right]$ & $34.22: 41.45: 52.44$ & $1.752 \pm 0.012$ & $30.11: 37.40: 48.98$ & $1.744 \pm 0.016$ \\
Inverse of the semimajor axis & $48.28 \pm 7.91$ & $1140.2 \pm 8.0$ & $53.48 \pm 10.14$ & $1145.4 \pm 10.3$ \\
Estimated time of perihelion passage $\left[10^{6}\right.$ yr from now $]$ & $-4.22:-2.97:-2.26$ & $0.03270 \pm 0.00027$ & $-3.81:-2.54:-1.84$ & $0.03252 \pm 0.00035$ \\
Percentage of dynamically old $\operatorname{VCs}(q<10 \mathrm{au})$ & $97 \%$ & $100 \%$ & $97 \%$ & $100 \%$ \\
\hline
\end{tabular}

Notes. Inverse semimajor axis values are in units of $10^{-6} \mathrm{au}^{-1}$. Orbital elements are described either by a mean value (with a $1 \sigma$ uncertainty) when a normal distribution is applicable, or otherwise by three deciles at 10, 50 (median), and $90 \%$.
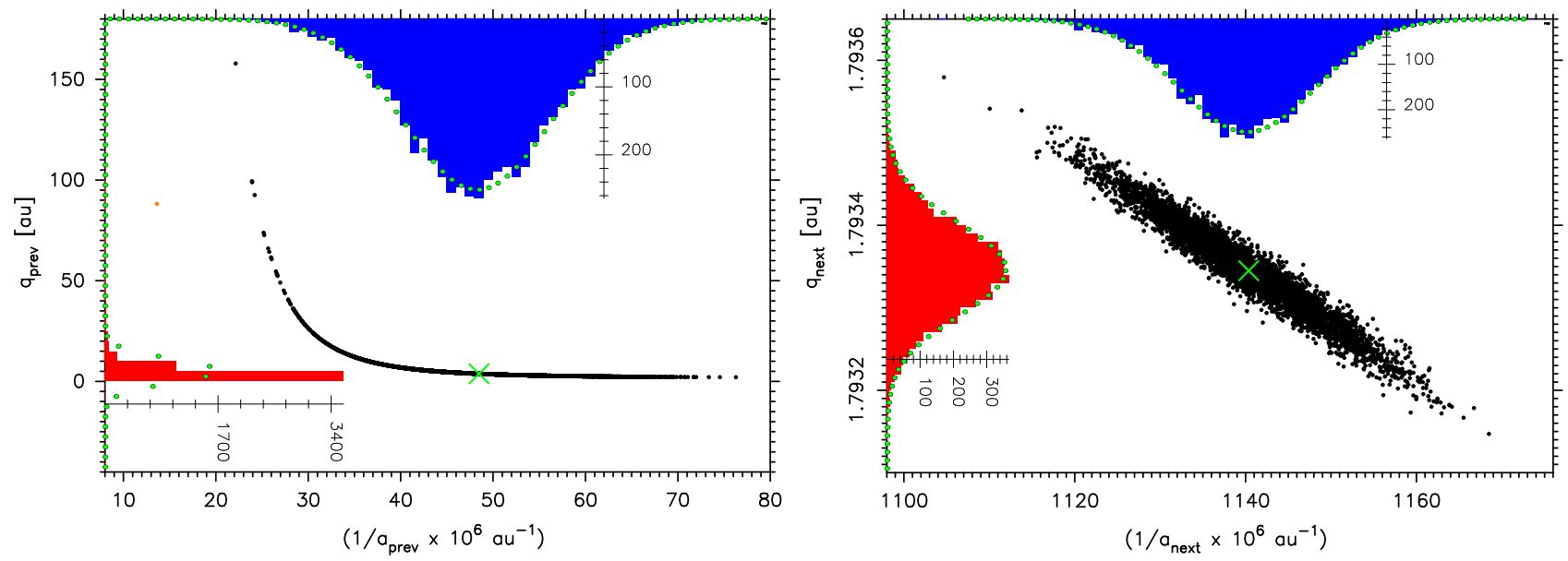

Fig. 2. Projection of $5001 \mathrm{VCs}$ of $\mathrm{K} 2$ onto the $1 / a-q$ plane (for the basic solution A1) augmented with two marginal distributions of these parameters. Left panel: orbital elements for $\mathrm{K} 2$ in the previous perihelion. Right panel: orbital elements in the subsequent perihelion. In each picture, the black point depicting the evolved nominal orbit is replaced by a green cross, and full green dots show a Gaussian fitting to $1 / a$ and $q$ marginal distributions. 

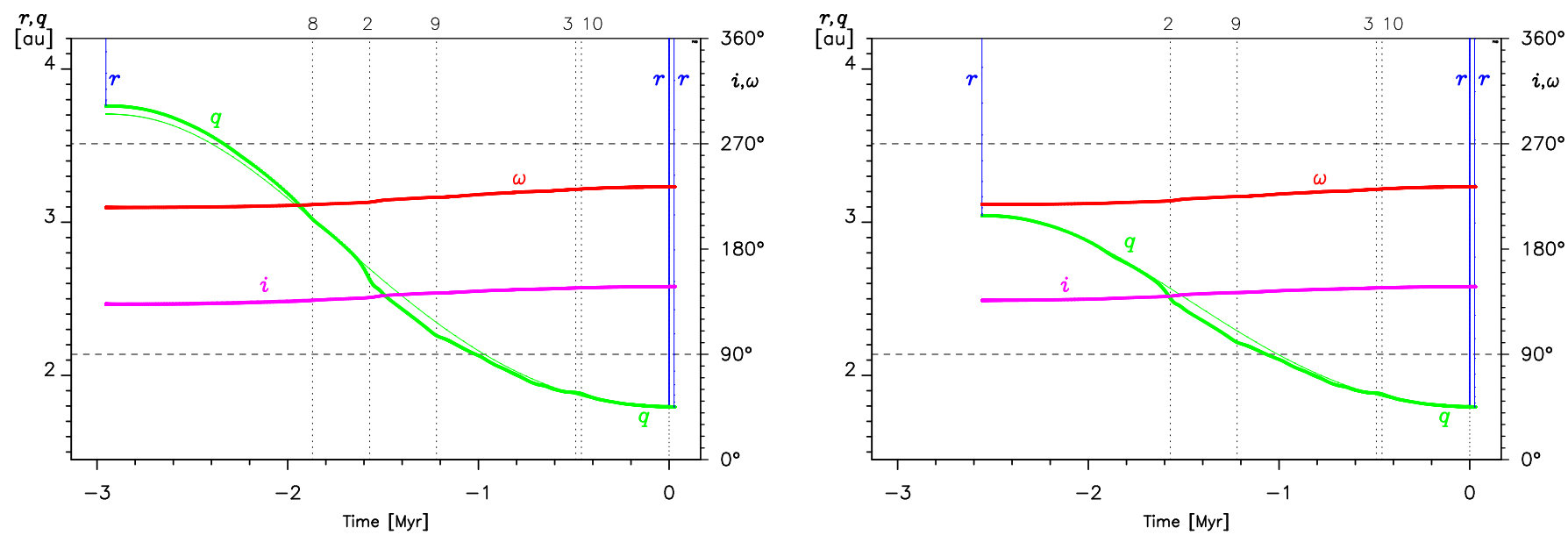

Fig. 3. Past and future dynamics of $\mathrm{K} 2$ for solutions $\mathrm{A} 1$ (left panel) and $\mathrm{A} 0$ (right panel). In both panels the horizontal time axis extends from the previous perihelion through the observed one to the subsequent perihelion passage. See text for a detailed explanation.

Table 4. Ten potentially strongest stellar perturbers of $\mathrm{C} / 2017 \mathrm{~K} 2$ in order of their increasing comet-star minimal distances.

\begin{tabular}{cclcccr}
\hline \hline & Star name & $M_{*}$ & $\gamma$ & $d_{\min }$ & $T_{\min }$ & \multicolumn{1}{c}{$V_{\text {rel }}$} \\
\hline 1 & GJ 217.1 & 2.4 & 0.239 & 1.31 & -0.85 & 7.65 \\
2 & HIP 30344 & 0.79 & 0.056 & 1.31 & -1.57 & 10.71 \\
3 & GJ 208 & 0.51 & 0.040 & 1.41 & -0.49 & 9.02 \\
4 & HD 49015 & 1.4 & 0.029 & 1.55 & -1.52 & 31.05 \\
5 & GJ 1049 & 0.51 & 0.016 & 1.56 & -0.62 & 20.34 \\
6 & HD 43947 & 1.2 & 0.026 & 1.75 & -0.66 & 26.41 \\
7 & HIP 27887 & 0.74 & 0.014 & 1.98 & -0.40 & 26.16 \\
8 & HD 37594 & 1.7 & 0.117 & 2.02 & -1.87 & 7.19 \\
9 & HD 54958 & 1.5 & 0.032 & 2.07 & -1.22 & 22.84 \\
10 & GJ 120.1ABC & 1.48 & 0.022 & 2.12 & -0.47 & 31.45 \\
\hline
\end{tabular}

Notes. For each star we present their estimated mass $M_{*}$ in solar masses, minimum distance $d_{\min }$ between a comet and a star in parsecs, an epoch $T_{\min }$ of this approach in Myrs and a relative velocity $V_{\text {rel }}$ in $\mathrm{km} \mathrm{s}^{-1}$. Parameter $\gamma=M_{*} /\left(d_{\min } \cdot V_{\text {rel }}\right)$ is the theoretical approximate measure of the stellar perturbation strength but the actual perturbation also strongly depends on the geometry of the encounter.

( $\omega$, red lines). Due to the invisible differences in $i$ and $\omega$ thin and thick lines overlap. Both these angular elements are expressed in a Galactic reference frame. Horizontal dashed lines call attention to the beginning of the second and fourth quarter of $\omega$, whose values $\left(90^{\circ}\right.$ and $\left.270^{\circ}\right)$ are important from the point of view of the Galactic disk perturbations. Vertical dotted lines mark the epochs of the closest comet-star encounters with star numbers from Table 4 at the top of the panels. Not all stars from Table 4 are indicated because the actual perturbation depends strongly also on the geometry of the approach. In the two figures we compare the results obtained from a full force model (Galactic and stellar perturbations, thick lines) with a simplified one, where stellar perturbations are omitted (thin lines).

It can be seen in Fig. 3 that K2 is now observed during its slowly decreasing phase of a perihelion distance evolution under the influence of a Galactic tide. In both our solutions its most probable aphelion distance is rather small (41 $500 \mathrm{au}$ and $37400 \mathrm{au}$, respectively) which makes this comet practically immune to any stellar perturbations. One can observe only small traces of interaction with HIP 30344, HD 54958, GJ 208 and GJ 120.1 ABC stellar system during the last orbital period. Moreover, our extended calculations show that, assuming no significant planetary perturbations occurred during the previous perihelion passage, its last but one approach to the Sun $6 \mathrm{Myr}$ ago also took place at a small heliocentric distance of a few au.

According to current data $\mathrm{K} 2$ will be significantly perturbed by planets and as a result its semimajor axis will be shortened down to about 875 au which will completely switch off Galactic and stellar perturbations during its future orbital period which will last about 32500 yr. Please keep in mind that non-gravitational effects due to a cometary activity near the perihelion, as well as violent changes in its behavior can modify this prediction.

Acknowledgements. We wish to thank an anonymous referee for valuable comments and suggestions. This research was partially supported by the project 2015/17/B/ST9/01790 founded by the National Science Center in Poland.

\section{References}

Gaia Collaboration (Prusti, T., et al.) 2016, A\&A, 595, A1

Hui, M.-T., Jewitt, D., \& Clark, D. 2018, AJ, 155, 25

Królikowska, M., \& Dybczyński, P. A. 2010. MNRAS, 404, 1886 Królikowska, M., \& Dybczyński, P. A. 2017, MNRAS, 472, 4634 Kunder, A., Kordopatis, G., Steinmetz, M., et al. 2017, AJ, 153, 75 Marsden, B. G., Sekanina, Z., \& Yeomans, D. K. 1973, AJ, 78, 211 Sitarski, G. 1989, Acta Astron., 39, 345

Sitarski, G. 1998, Acta Astron., 48, 547 